\title{
Gasos Guínicos \\ Extirpación quirúrgica con cierre primario y uso de Isotretinoína en Acné queloídeo de la nuca. Serie de 6 casos clínicos
}

\author{
Mauricio Sandoval-Osses ${ }^{1}$, María Jesús Rojas-Lechuga², María Carolina González-Aspillaga²
}

\section{RESUMEN}

El Acné queloídeo de la nuca se presenta principalmente en hombres jóvenes de raza negra; su etiología es desconocida, pero se piensa que sería multifactorial. El cuadro clínico corresponde a pápulas y pústulas que tienden a confluir en placas de aspecto queloídeo y que se observan como alopecia cicatricial, produciendo gran repercusión tanto física como psicológica en el paciente. Histológicamente se distingue una etapa temprana y una avanzada. No existe tratamiento curativo para este cuadro, pero se ha experimentado con múltiples opciones de tratamiento. En este trabajo se exponen los resultados de nuestra experiencia terapéutica con cirugía excisional asociada a cierre primario y posterior uso de Isotretinoína oral.

Palabras claves: Acné queloideo de la nuca; cirugía; Isotretinoína.

\section{SUMMARY}

The Acne keloidalis nuchae occurs mainly in young black men; its etiology is unknown but is thought to be multifactorial. Clinically it presents as papules and pustules which tend to coalesce in keloid-like plaques and they are observed as scarring alopecia, producing great impact both physically and psychologically in the patient. An early and an advanced stage can be distinguished with histological techniques. There is no cure for this pathology, even though multiple treatments have been tried. In this paper, we expose the results of our therapeutic experience with excisional surgery associated with primary closure and subsequent use of oral Isotretinoin.

Key words: Acne keloidalis nuchae; surgery; Isotretinoin.
$\mathrm{E}$ l Acné queloídeo de la nuca (AQN) fue primariamente descrito por Kaposi en el año 1869 como Capillitii papillaris dermatitis. Tres años después Bazin lo llamó Acné queloídeo, sin embargo, esta condición no corresponde a un cuadro de acné ni a un queloide. A lo largo del tiempo, esta patología ha aparecido en la literatura bajo una variedad de nombres: foliculitis keloidalis, sicosis nuchae, foliculitis queloide, liquen nucal keloidalis y foliculitis scleroticans nucal ${ }^{1}$.

El AQN se presenta principalmente en hombres jóvenes y de raza negra, su patogenia es desconocida, pero se sospecha que sería multifactorial ${ }^{2}$. Actualmente es considerado dentro del grupo de las alopecias cicatriciales primarias $^{3}$.
A continuación, mostraremos nuestra experiencia en el tratamiento de AQN con cirugía excisional y cierre primario (CECP) seguido por tratamiento con Isotretinoína oral.

El proceso consiste en delimitar el área afectada, se anestesia esta área con lidocaína al $2 \%$ con vasoconstrictor, luego se aplica anestesia tumescente con solución de Klein en toda la zona de la nuca. Se extirpa el área demarcada con una incisión elíptica hasta el plano hipodérmico, y se envía el tejido extirpado a anatomía patológica. Se realiza hemostasia con electrocoagulador y ligadura de vasos de mayor calibre, luego se realiza una liberación extensa entre los planos dérmicos e hipodérmicos, teniendo especial cuidado en realizar una buena hemostasia de las arteriolas perforantes en la región oc-

${ }^{1}$ Departamento de Dermatología. Facultad de Medicina. Pontificia Universidad Católica de Chile. Santiago, Chile. ${ }^{2}$ Facultad de Medicina. Pontificia Universidad Católica de Chile. Santiago, Chile. 
cipital. Se sutura en dos planos, con Catgut 2.0 el plano hipodérmico y con puntos Donati vertical con seda 2.0 el plano dermo-epidérmico, logrando un cierre primario total del defecto. Se cubre el área con apósitos y se aplica collar cervical rígido por una semana. A los 7 días, se retira el collar cervical y se realiza una curación, a los 14 días se retiran las suturas. Se indicó en todos los casos cefadroxilo $500 \mathrm{mg}$ cada 12 horas por 7 días como profilaxis antibiótica y ketoprofeno $100 \mathrm{mg}$ cada 12 horas por 5 días como analgesia. A los 30 días de la cirugía se inició isotretinoína oral $20 \mathrm{mg}$ al día hasta completar una dosis total de $120 \mathrm{mg} / \mathrm{kg}$.

\section{Gasos Guínicos}

Se incluyeron 6 pacientes (Tabla 1), la edad promedio al momento de la cirugía fue de 27 años (21-35 años), la edad promedio al diagnóstico de AQN fue de 17 años (13 años-19 años), con una relación masculino:femenino de $5: 1$.

En relación a los tratamientos previos, todos fueron tratados por distintos períodos de tiempo con: clindamicina tópica, doxiciclina oral, tetraciclina oral, en 3 casos con isotretinoína, pero ningún tratamiento fue exitoso.

Durante la cirugía, no hubo problemas en controlar la hemostasia, ni otros inconvenientes. El procedimiento fue bien tolerado y la mayoría, presentó dolor postoperatorio leve manejado con antiinflamatorios no esteroideos. En ninguno de los casos hubo infección ni hematomas postoperatorios. Se recomendó no realizar actividad física ni laboral hasta el retiro de puntos.

Uno de los 6 casos evolucionó con una cicatriz hipertrófica a los 6 meses de seguimiento, fue tratado con corti- coides intralesionales con buena respuesta.

El seguimiento promedio sin recurrencias en nuestra serie fue de 4 años (3-6 años)

\section{Discusión}

El AQN se presenta principalmente en hombres jóvenes y de raza negra, es 20 veces más frecuente en hombres que en mujeres y su desarrollo antes de la pubertad o después de los 50 años es poco frecuente ${ }^{1,2}$.

Su patogenia es desconocida ${ }^{3}$, sin embargo, Ogunbiyi A. et al encontró que un 15\% de sus pacientes con AQN tenían antecedentes familiares de esta misma patología. En la literatura se han descrito casos familiares; en un caso se ven involucrados un padre y sus tres hijos, pero no sus dos hijas², por lo que se cree que existirían variables genéticas involucradas. También existirían factores traumáticos; es así como el roce constante de la camisa o un corte de pelo muy corto (especialmente el rizado, ya que la curvatura del folículo piloso genera impacto del pelo en la piel al crecer) pueden ser un factor precipitante. Además, se han establecido otras asociaciones; existen casos vinculados al uso de ciclosporina, difenilhidantoína y carbamazepina, en los cuales las lesiones se resolvieron cuando las drogas fueron suspendidas. También fue publicada una serie de 4 casos asociados a acantosis nigricans y síndrome metabólico. Debido a la serie de factores descritos, todo indica que la etiología sería multifactorial ${ }^{4,5}$.

Clínicamente, el AQN se presenta como pápulas de 2 a $4 \mathrm{~mm}$, con pústulas en menor proporción, que forman placas de aspecto queloídeo, generalmente dolorosas y que en la mayoría de los casos presentan una secreción

Tabla 1

Identificación de pacientes.

\begin{tabular}{llllll}
\hline $\begin{array}{l}\text { Número } \\
\text { de caso }\end{array}$ & Sexo & $\begin{array}{l}\text { Edad al } \\
\text { diagnóstico AQN }\end{array}$ & $\begin{array}{l}\text { Edad al momento } \\
\text { de la cirugía }\end{array}$ & $\begin{array}{l}\text { Tiempo de } \\
\text { seguimiento }\end{array}$ & Recurrencias \\
\hline 1 (Fig. 1) & Masculino & 13 años & 21 años & 4 años & No \\
2 (Fig. 2) & Femenino & 18 años & 35 años & 6 años & No \\
3 (Fig. 3) & Masculino & 17 años & 23 años & 3 años & No \\
4 (Fig. 4) & Masculino & 19 años & 29 años & 3 años & No \\
5 (Fig. 5) & Masculino & 18 años & 26 años & 4 años & No \\
6 (Fig. 6) & Masculino & 18 años & 28 años & 4 años & No \\
\hline
\end{tabular}




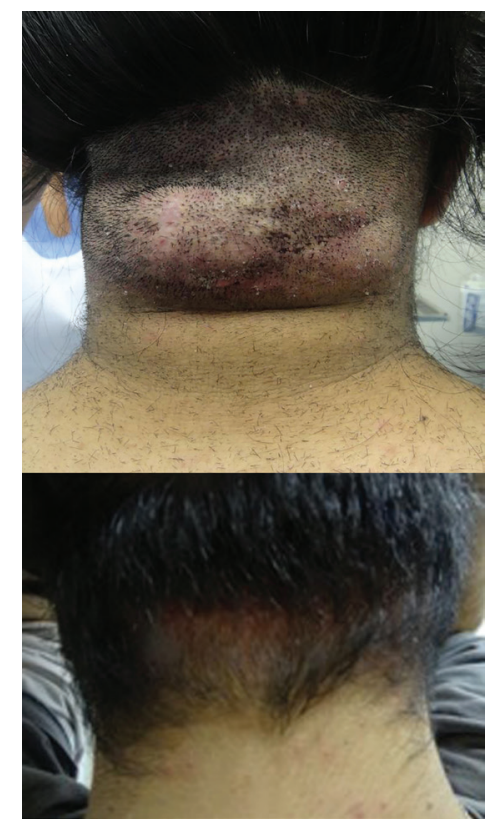

Figura 1

Caso 1. Antes de la cirugía y luego de 4 años de seguimiento.

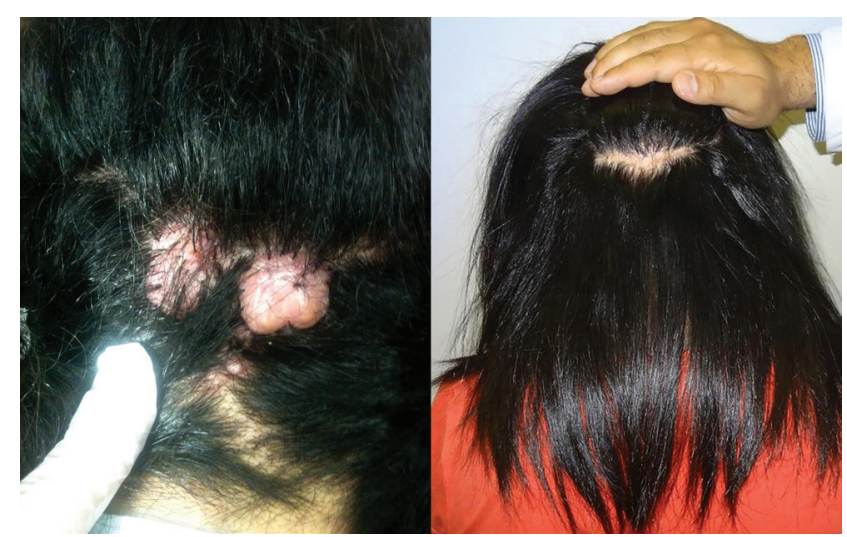

Figura 2

Caso 2. Antes de la cirugía y seis años post tratamiento y uso de Isotretinoína.

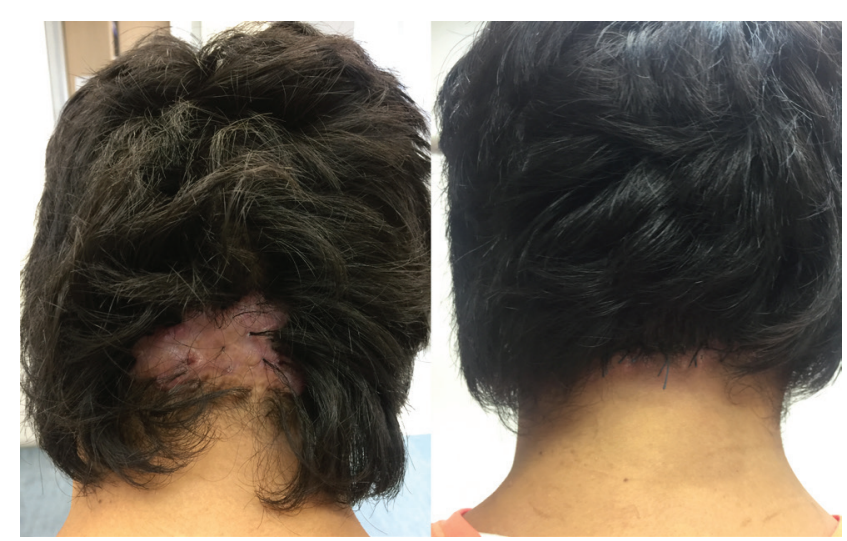

Figura 3

Caso 3. Antes de la cirugía y post tratamiento a los 3 años de seguimiento

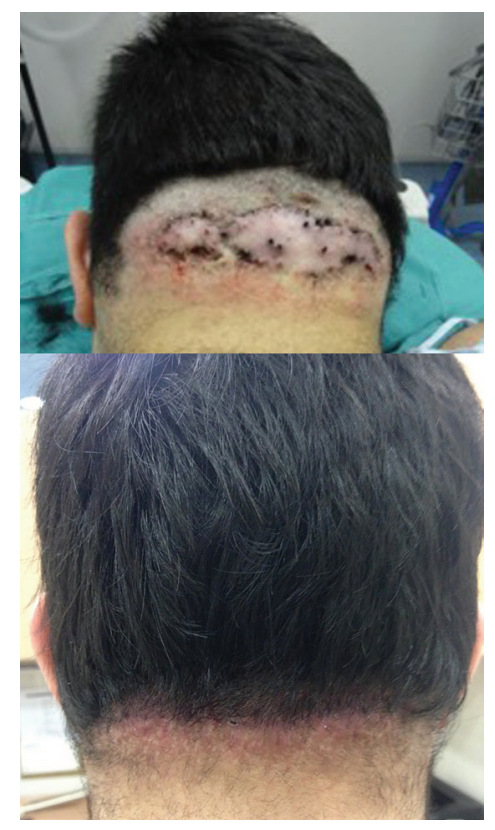

\section{Figura 4}

Caso 4. Antes de la cirugía y tres años posterior a la cirugía y uso de isotretinoína
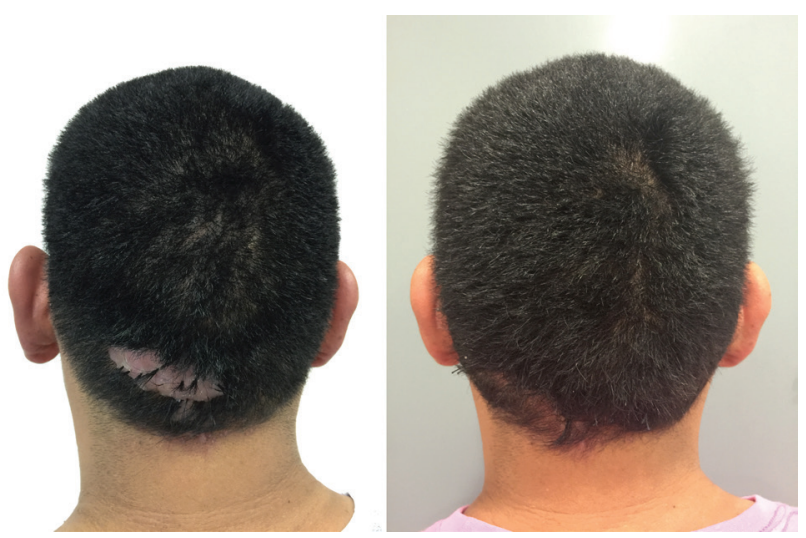

Figura 5

Caso 5. Antes de la cirugía y post tratamiento a los 4 años de seguimiento

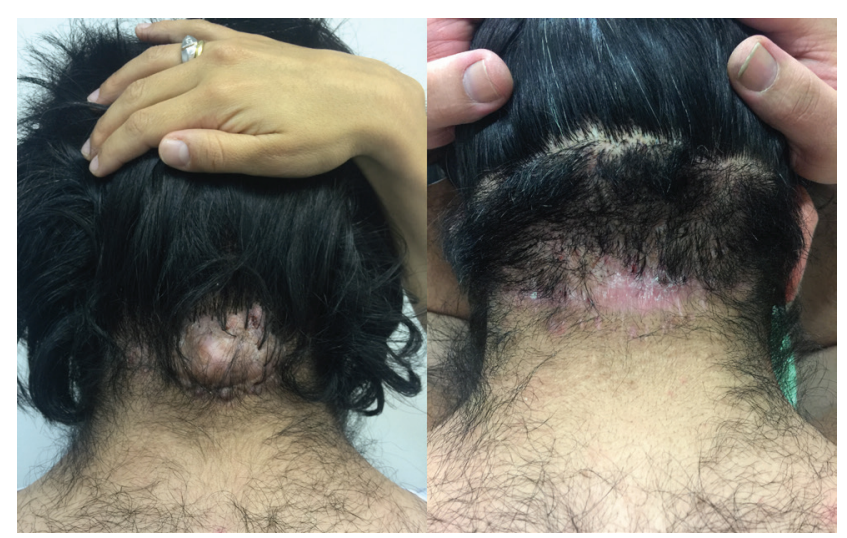

\section{Figura 6}

Caso 6. Antes de la cirugía y post tratamiento a los 4 años de seguimiento 
de mal olor. No se observan comedones. La enfermedad conduce a una alopecia cicatricial y las lesiones crónicas contienen abscesos y fístulas. El cuadro causa gran estrés y mala calidad de vida en el paciente ${ }^{2}$.

Histológicamente, se caracteriza por glándulas sebáceas marcadamente disminuidas o ausentes. En la etapa inicial se observa inflamación folicular y perifolicular, mientras que en etapas más tardías los folículos pilosos se rompen y se observan fragmentos de pelo fracturado rodeado por inflamación granulomatosa, generándose fibrosis dérmica y convirtiendo las lesiones en tejido cicatricial $^{2}$.

No existe un buen tratamiento curativo descrito para el AQN, por lo que es esencial la prevención; la cual podría consistir principalmente en un corte de pelo adecuado y en no usar camisas ajustadas en la zona del cuello. Se han probado diferentes tratamientos con variados grados de efectividad, entre ellos corticoides tópicos, corticoides intralesionales, retinoides tópicos, antibióticos tópicos como clindamicina y eritromicina, antibióticos orales (Tetraciclinas), imiquimod, 5-fluorouracilo, láser (dióxido de carbono o Nd: YAG), expansión de tejido, radioterapia, criocirugía, electrocirugía y cirugía6; pero ninguno de los tratamientos mencionados ha generado un aclaramiento total de las lesiones, ni la ausencia de recurrencia de éstas. Entre las opciones quirúrgicas se han planteado distintas modalidades; cierre primario, cierre por segunda intención e injerto. Los corticoides orales serían eficaces en detener la progresión, pero al suspenderlos, el cuadro recurriría ${ }^{7}$. Existe un reporte que muestra mejoría importante de un caso con el uso de isotretinoína ${ }^{8}$.

En un trabajo de Gloster H et al. se muestran los resultados del manejo quirúrgico de AQN en 25 pacientes; en los que se practicó CECP en una etapa en 20 pacientes, CECP en 2 etapas en 4 pacientes y cirugía con cierre por segunda intención en un paciente; obteniéndose buenos resultados postoperatorios, pero al cabo de un tiempo, 15 pacientes desarrollaron pequeñas pústulas y pápulas en la zona de la cicatriz operatoria y 5 pacientes desarrollaron cicatrices hipertróficas que requirieron manejo con corticoides tópicos o intralesionales ${ }^{9}$. Respecto a la cirugía con cierre por segunda intención se han publicado múltiples trabajos; entre ellos destaca Califano J et al, que describe 5 pacientes en los que no recurre el cuadro de AQN, pero presentan regulares a malos resultados estéticos (alopecia y discromía postinflamatoria)10. Otros autores han referido mejores resultados estéticos con cierre por segunda intención, pero con un número menor de pacientes ${ }^{11,12,13}$.

Recientemente, Maranda et al, realizaron una revisión sistemática de la literatura respecto al tratamiento del AQN, concluyendo que el tratamiento médico puede ser usado en casos moderados y la extirpación quirúrgica debe ser reservada para lesiones extensas con fibrosis prominente. Además, considera que el tratamiento con láser con 1064nm Nd:YAG, 810nm Diodo y láser $\mathrm{CO}_{2}$ pueden ser una alternativa de tratamiento. Sin embargo, concluyen que los estudios de la revisión, incluyen series de casos y estudios pequeños, por lo que mayores estudios randomizados se requieren para evaluar la real efectividad de estos tratamientos ${ }^{14}$.

En nuestra serie de casos, hemos encontrado en promedio, un tiempo de evolución del AQN previo a la cirugía de 9,8 años y una edad promedio de 27 años al momento de la intervención. Durante el procedimiento, no se presentaron problemas al controlar la hemostasia, aun cuando la zona occipital tiene vasos arteriales de mediano calibre y alto flujo, lo que puede deberse a la meticulosa hemostasia de la zona operatoria. Respecto a esta buena evolución, creemos que es muy relevante que se trata de pacientes jóvenes y sin patologías asociadas.

En todos estos casos tratados con el uso de CECP e isotretinoína oral, se obtuvo una mejoría de la enfermedad sin recurrencia actual con un seguimiento de entre 3 y 6 años. Desconocemos el mecanismo mediante el cual este tratamiento evita la recurrencia de las lesiones. Esta es la primera publicación conocida por nosotros que plantea esta combinación de tratamiento quirúrgico seguido del uso de isotretinoína oral como una alternativa terapéutica exitosa en el tratamiento del AQN.

\section{Conclusiones}

La CECP es una cirugía simple, ambulatoria y bien tolerada que en conjunto al uso postquirúrgico de isotretinoína oral, se presenta como una excelente alternativa en el tratamiento del AQN, generando una mejoría inmediata post cirugía y sin recurrencias a mediano plazo. El resultado de este tratamiento genera una mejoría sustancial en la calidad de vida del paciente. 


\section{REFERENCIAS}

1. Adegbidi H, Atadokpede F, do Ango-Padonou F, Yedomon H. Keloid acne of the neck: epidemiological studies over 10 years. Int J Dermatol 2005; 44(1):49-50

2. Ogunbiyi A, George A. Acne keloidalis in females: Case report and review of literature. J Natl Med Assoc 2005;97(5):736-8

3. Sperling L, Homoky C, Pratt L, Sau P. Acne keloidalis is a form of primary scarring alopecia. Arch Dermatol 2000; 136:479-84

4. Verma SB, Wollina U. Acne keloidalis nuchae: another cutaneous symptom of metabolic syndrome, truncal obesity, and impending/ overt diabetes mellitus?. Am J Clin Dermatol 2010;1 1(6):433-6

5. Piaserico S, Fortina AB, Cavallini F, Alaibac M. Acne Keloidalis of the Scalp in a Renal Transplant Patient Treated with Cyclosporine. Acta Derm Venereol 2009;89(3):312-3

6. Esmat SM, Abdel Hay RM, Abu Zeid O, Hosni HN. The efficacy of laser-assisted hair removal in the treatment of acne keloidalis nuchae; a pilot study. EurJ Dermatol 2012;22(5):645-50

7. Alexis A, Heath CR, Halder RM. Folliculitis Keloidalis Nuchae and Pseudofolliculitis Barbae: Are Prevention and Effective Treatment Within Reach?. Dermatol Clin 2014;32(2):183-91
8. Stieler W, Senff H, Jänner M. Folliculitis nuchae scleroticanssuccessful treatment with 13-cis-retinoic acid (isotretinoin). Hautarzt 1988;39(11):739-42.

9. Gloster H. The surgical management of extensive cases of acne keloidalis nuchae. Arch dermatol 2000;136: 1376-9

10. CalifanoJ, Miller S, Frodel J. Treatment of Occipital Acne Keloidalis by Excision Followed by Secondary Intention Healing.Arch Facial Plast Surg 1999;1:308-11

11. Etzkorn JR, Chitwood K, Cohen G. Tumor stage acne keloidalis nuchae treated with surgical excision and secondary intention healing. J Drugs Dermatol. 2012;11(4):540-1

12. Beckett N, Lawson C, Cohen G. Electrosurgical excision of acne keloidalis nuchae with secondary intention healing. J Clin Aesthet Dermatol. 201 1;4(1):36-9

13. Bajaj V, Langtry JA. Surgical excision of acne keloidalis nuchae with secondary intention healing. Clin Exp Dermatol. 2008;33(1):53-5

14. Maranda EL, Simmons BJ, Nguyen AH, Lim VM, Keri JE. Treatment of Acne Keloidalis Nuchae: A Systematic Review of the Literature. Dermatol Ther (Heidelb). 2016;6(3):363-78 